\title{
Herstellung und Charakterisierung von flexiblen potentiometrischen pH-Sensoren auf Parylene C Substraten
}

\author{
Franz Selbmann ${ }^{1,2}$, Frederic Güth ${ }^{1}$ Christian Miersch ${ }^{1}$ und Yvonne Joseph ${ }^{1}$ \\ ${ }^{1}$ Institut für Elektronik- und Sensormaterialien, TU Bergakademie Freiberg, Freiberg, Deutschland \\ ${ }^{2}$ Fraunhofer-Institut für Elektronische Nanosysteme, Chemnitz, Deutschland \\ Kontakt: franz.selbmann@esm.tu-freiberg.de
}

\section{Einleitung}

Sensoren sind ein Schlüsselelement, um intelligente Systeme und das Internet der Dinge (engl. Internet of Things IoT) zu ermöglichen. Für die Realisierung smarter medizinischer Wearables, intelligenter Klebebänder oder für die Integration von Sensoren zur Überwachung von Leichtbaustrukturen werden dabei insbesondere Sensorkonzepte auf Basis flexibler Materialien benötigt. Dabei wurde in der Vergangenheit eine Vielzahl an Sensorkonzepten für die Messung physikalischer Größen von starren Substraten auf flexible übertragen oder speziell für letztere entwickelt. Im Gegensatz dazu gibt es bisher nur wenige flexible Sensorkonzepte für die Messung chemischer Größen.

Der pH-Wert als Maß für die Aktivität der Hydroniumionen ist eine wichtige Größe für viele industriell oder auch biologisch relevante, chemische Reaktionen, sodass ein entsprechend hohes Interesse für dessen Messung besteht. Um den pH-Wert im Rahmen von Anwendungen wie bspw. medizinischen Wearables, der Strukturüberwachung von Leichtbauteilen oder mittels geometrieadaptiver Sensorik messen zu können, ist ein Konzept für einen flexiblen $\mathrm{pH}$ Sensor mit gleichzeitig hohem Miniaturisierungspotenzial zielführend. Letzteres ermöglicht darüber hinaus durch die damit einhergehende erhöhte Integrationsdichte sowie besseres Handling auch eine Weiterentwicklung für bereits etablierte Anwendungen der pH-Sensorik, bspw. im Bereich der Umwelt- oder Trinkwasserüberwachung. Weitere Anforderungen an einen derartigen flexiblen $\mathrm{pH}$-Sensor sind daher der Einsatz chemisch stabiler Materialien, die weiterhin mit den verwendeten Herstellungsprozessen kompatibel sind; ein einfaches und zuverlässiges, kostengünstiges sowie gleichzeitig im Hinblick auf die Massenfertigung skalierbares Sensorprinzip; hohe mechanische Festigkeiten und Zuverlässigkeiten bei gleichzeitig geringen Schichtdicken und hohen Flexibilitäten; sowie die möglichst ausschließliche Verwendung biokompatibler Materialien.

Im Rahmen der vorliegenden Arbeit soll daher ein neuartiges Konzept für einen flexiblen, ultra-dünnen $\mathrm{pH}$-Sensor beschrieben werden, der die o. g. Eigenschaften abbildet.

\section{Methoden und Materialien}

Der entwickelte flexible $\mathrm{pH}$-Sensors wurde unter Nutzung des potentiometrischen Funktionsprinzips mit einer Arbeitselektrode aus Iridiumoxid und einer Referenzelektrode aus Silber/Silberchlorid aufgebaut. Die Aktivität der Hydroniumionen wird dabei entsprechend der Nernst-Gleichung in ein elektrisches Potenzial E umgesetzt.
In Folge seiner chemischen Beständigkeit gegenüber allen gängigen Säuren, Basen und Lösemitteln sowie der sich daraus ergebenden Kompatibilität mit verschiedenen Technologien zur Schichtabscheidung und -strukturierung wurde Parylene als Material für das flexible Substrat gewählt. Poly-p-xylylen (Parylene) beschreibt eine thermoplastische Polymerfamilie, die eine einzigartige Kombination exzellenter Eigenschaften auszeichnet: Chemische Inertheit, Biostabilität und Biokompatibilität (nach ISO 10993), optische Transparenz, geringe Permeabilität gegenüber Wasser und Gasen sowie eine für Polymere vergleichsweise hohe thermische Beständigkeit. Gleichzeitig wird Parylene aus der Gasphase bei Raumtemperatur konform abgeschieden, was den Eintrag intrinsischer mechanischer Schichtspannungen vermeidet, sodass sich die hergestellten flexiblen Parylene-Substrate nicht aufrollen [1,2]. Neben Anwendungen im Bereich der Verkapselung kann Parylene aufgrund seiner Kompatibilität mit etablierten Mikrotechnologien u. a. zur Realisierung ultra-dünner flexibler Leiterplatten eingesetzt werden [3]. Parylene eignet sich daher nicht nur als Substrat für flexible Sensorik, sondern bietet darüber hinaus eine Packaging-Plattform für die direkte Integration von benötigter Elektronik zur Datenaufbereitung und -kommunikation neben dem eigentlichen Sensorelement.

Der für die Herstellung des flexiblen potentiometrische $\mathrm{pH}-$ Sensors verwendete Prozess ist in Abbildung 1 schematisch dargestellt: Durch Vereinzeln eines 6"-SiliziumWafers mittels Sägen wurden Chips gewonnen (1), auf die anschließend eine wasserlösliche Opferschicht pipettiert bzw. mittels Spincoating abgeschieden und getrocknet wurde (2). Im nächsten Schritt wurde eine etwa $10 \mu \mathrm{m}$ dicke Schicht Parylene C aus der Gasphase mit Hilfe eines Plasma Parylene LC 300 RW (Plasma Parylene Systems $\mathrm{GmbH}$ ) abgeschieden (3). Unter Verwendung einer entsprechenden Edelstahl-Schattenmaske wurden danach die Titan-Elektroden paarweise aufgesputtert (4). Anschließend wurde eine der Titanelektroden durch Cyclovoltammetrie mit Iridiumoxid beschichtet, um die Arbeitselektrode zu realisieren (5).

Die Cyclovoltammetrie wurde in einer Drei-ElektrodenKonfiguration (Referenzelektrode: $3 \mathrm{M} \mathrm{KCl} \mathrm{Ag/AgCl,} \mathrm{Ge-}$ genelektrode: Platinnetz) durchgeführt. Als Iridiumpräkursor wurde ein Iridium-Oxalat-Komplex verwendet. Dieser wurde in wässriger Lösung durch die Reaktion von Iridiumtetrachlorid-Hydrat als Iridiumquelle, Kaliumoxalat-Monohydrat als Komplexbildner, Kaliumcarbonat zur Einstellung 


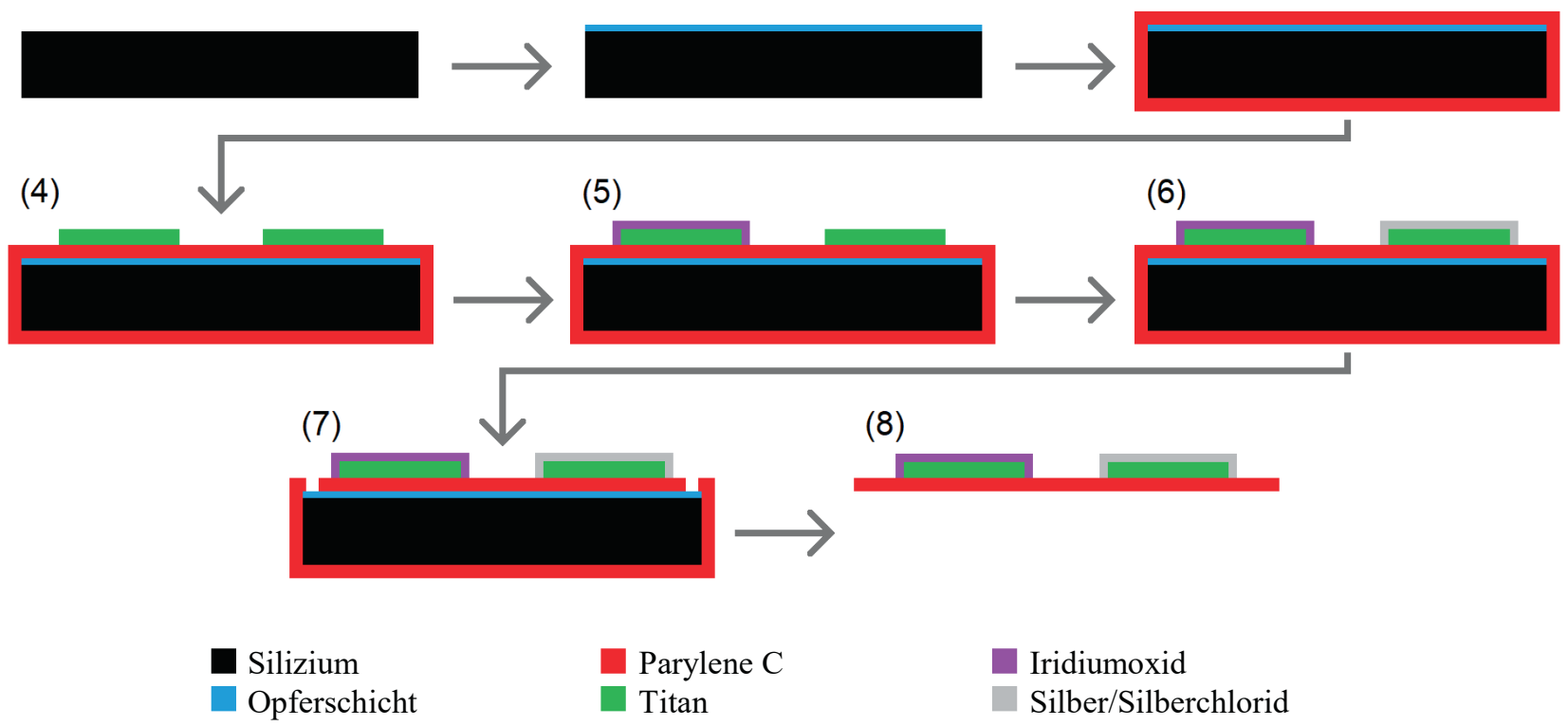

Abb. 1: Schematische Darstellung des Prozesses für die Herstellung der flexiblen potentiometrischen pH-Sensoren auf Basis von Parylene.

des $\mathrm{pH}$-Wertes sowie Wasserstoffperoxid zur Beschleunigung der Komplexbildung hergestellt [4].

Zur Abscheidung wurde das Potential an der Arbeitselektrode mehrfach mit einer Geschwindigkeit von $0,1 \mathrm{~V} / \mathrm{s}$ zwischen den Grenzen von -0,7 V und 0,9 V variiert. Zur Durchführung der Abscheidung und der elektrochemischen Experimente wurde ein PalmSens4 Potentiostat (PalmSens) verwendet [5].

Auf den flexiblen $\mathrm{pH}$-Sensor wurde durch Aufrakeln einer Silber/Silberchlorid-Paste (Gwent Electronic Materials, $\mathrm{Ag} / \mathrm{AgCl}$ 60/40) über die zweite Titanelektrode eine QuasiReferenzelektrode (QRE) realisiert (6). Die $\mathrm{Ag} / \mathrm{AgCl}$ Schicht wurde anschließend bei $60^{\circ} \mathrm{C}$ für 30 Minuten in einem Trockenschrank behandelt.

In Vorbereitung des Freistellens des flexiblen ParyleneSubstrats wurde das Parylene am Probenrand mit Hilfe eines Skalpels eingeschnitten, um einen entsprechenden Zugang zur Opferschicht zu realisieren (7), die dann im letzten Schritt in Wasser aufgelöst wurde (8). Ein Foto einer freigestellten Elektrode ist in Abb. 2 gezeigt.

Die hergestellten Iridiumoxidelektroden wurden mit Hilfe der Rasterelektronenmikroskopie (REM) untersucht, um Oberflächenbeschaffenheit, d. h. Rauheit und Homogenität der Schicht, zu beurteilen. Zur Bewertung der Grenzflächen und Schichtdicken wurden zudem Querschliffe im REM untersucht.

Die sensorischen Eigenschaften der hergestellten flexiblen $\mathrm{pH}$-Sensoren wurden durch die Bestimmung der Potentialdifferenz zwischen der IrOx-Elektrode und entweder der aufgerakelten QRE oder einer externen Referenzelektrode (Ag/AgCl $3 \mathrm{M} \mathrm{KCl})$ ermittelt. Als Messmedium wurden Pufferlösungen (BDH Chemicals) verwendet. Zusätzlich wurden Titrationsexperimente mit Hilfe einer Pufferlösung durchgeführt, die aus einer äquimolaren Mischung von jeweils 0,04 molarer Bor-, Phosphor- und Essigsäure bestand, zu der jeweils mit 1 molarer Salzsäure- bzw. Kaliumhydroxidlösung titriert wurde. Während der Titration wurde der pH-Wert mit einer gewöhnlichen $\mathrm{pH}$-Einstabmesskette (Mettler Toledo InLab Science) bestimmt. Alle Experimente wurden temperiert bei $(25 \pm 0,5){ }^{\circ} \mathrm{C}$ durchgeführt.

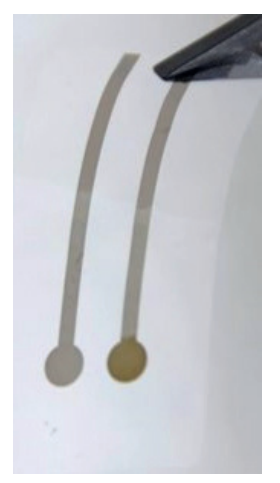

Abb. 2: Fotographische Aufnahme des freigestellten potentiometrischen $\mathrm{pH}-\mathrm{Sensors}$. Links) QRE, rechts) IrOxArbeitselektrode. 


\section{Ergebnisse und Diskussion}

Die Dicke der IrOx-Schicht kann während der Abscheidung durch die Anzahl der Wiederholungen der Potentialzyklen eingestellt werden. Dabei nimmt die Schichtdicke mit steigender Zyklenzahl zu. In Abbildung 3 sind REM-Querschnittsaufahmen der resultierenden Schichten bei verschiedenen Zyklenzahlen gezeigt. Als Optimum der untersuchten Parameter stellte sich eine Zyklenzahl von 100 mit einer resultierenden Schichtdicke von ca. $80 \mathrm{~nm}$ heraus, da hier eine dichte und homogene Schicht erzeugt wurde. Dickere Schichten zeigten häufig Risse und bei dünneren Schichten war nicht immer eine vollständige Beschichtung gegeben.

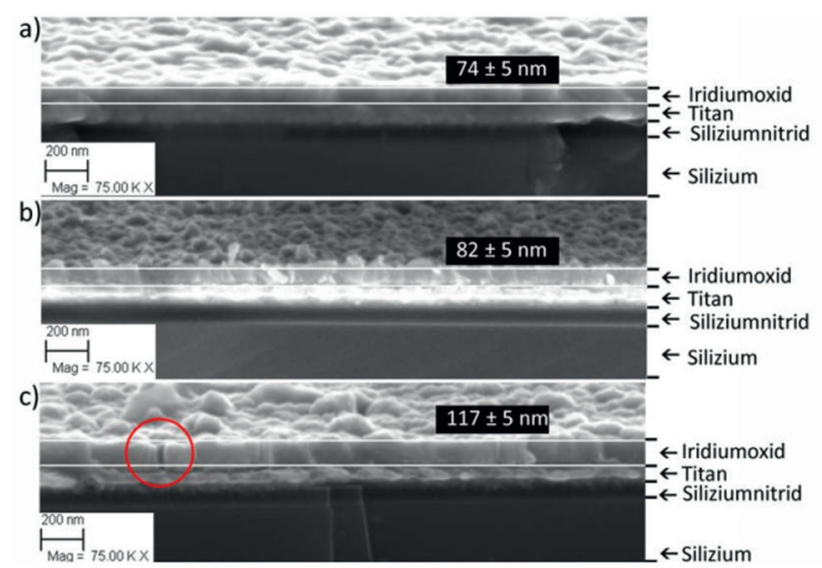

Abb. 3: REM-Aufnahmen von Probenquerschnitten hergestellter Elektroden. Die Dicke der IrOx-Schichten ist angegeben. Die Anzahl der durchlaufenen Zyklen während der Abscheidung war a) 50 Zyklen, b) 100 Zyklen, c) 200 Zyklen. In Abbildung c) ist ein Riss durch die Schicht markiert.

In Abbildung 4 sind die Ergebnisse eines Titrationsexperiments dargestellt. Die IrOx-Elektroden zeigen eine lineare Sensorantwort und eine $\mathrm{pH}$-Empfindlichkeit von etwa

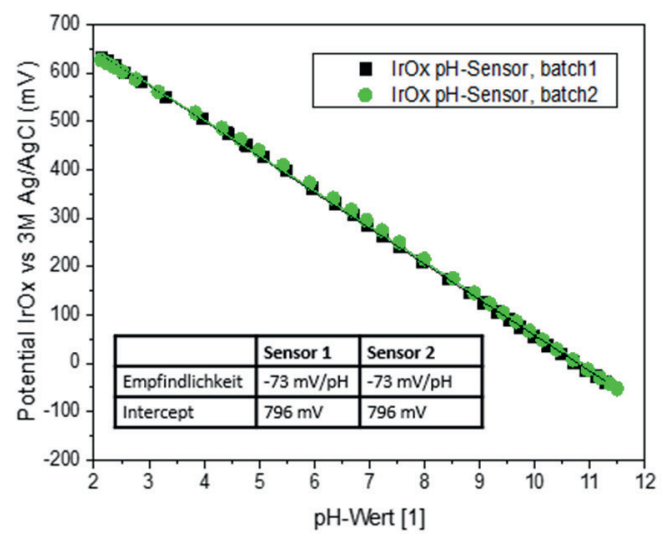

Abb. 4: Vergleich der pH-Abhängigkeit des Sensorsignals zweier unabhängig voneinander hergestellten $\mathrm{IrOx}-\mathrm{pH}-$ Elektroden gegenüber der kommerziellen Referenzelektrode.
- $73 \mathrm{mV} / \mathrm{pH}$ zwischen pH 2 und 11,5. Diese Werte sind vergleichbar mit veröffentlichten Ergebnissen [4]. Elektroden aus mehreren Chargen, die an verschiedenen Tagen hergestellt wurden, zeigen eine hohe Reproduzierbarkeit in Bezug auf Empfindlichkeit und Achsenabschnitt, wie die eng übereinanderliegenden Datenpunkte in Abbildung 4 belegen. Diese Ergebnisse lassen auf eine hohe Robustheit des Beschichtungsprozesses schließen.

Das Driftverhalten einer IrOx-Elektrode in einem pH 4 Puffer über 22 Stunden ist in Abb 5 dargestellt. Die Driftrate beträgt weniger als $1 \mathrm{mV} / \mathrm{h}$, was für viele Anwendung ausreichend ist. Es kann vermutet werden, dass die Drift durch parallel ablaufende Sekundärreaktionen an der Grenzfläche zwischen Messlösung und sensitiver Schicht verursacht wird. Möglicherweise können zukünftig elektrochemische Vorbehandlungen oder auch der Einsatz von protonenleitenden Schutzschichten, wie Nafion oder Tantalpentoxid die Stabilität des Messsignals verbessern.

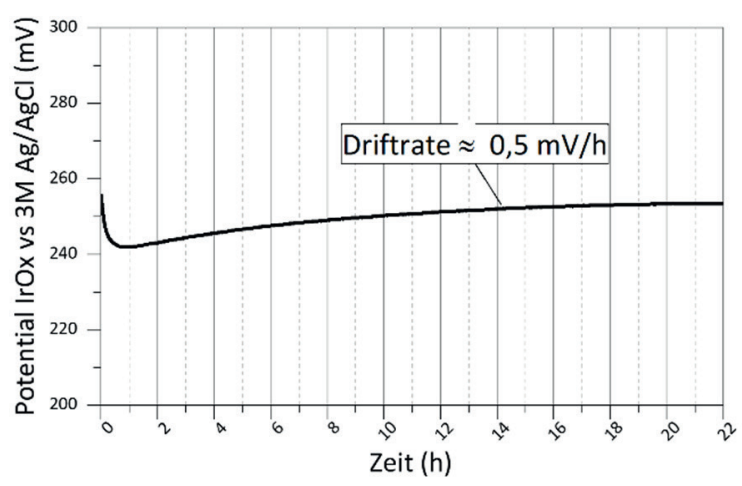

Abb. 5: Driftverhalten einer $\mathrm{pH}-\mathrm{IrOx}-$ Elektrode in einem pH 4 Puffer über 22 Stunden gegenüber der kommerziellen Referenzelektrode.

Zur Beurteilung der sensitiven Eigenschaften im gebogenen Zustand wurde eine Elektrode um einen zylindrischen Biegekörper mit einem Biegeradius von $4 \mathrm{~mm}$ gelegt und fixiert (siehe Abb. 6). Mit dieser Elektrode wurde dann ein weiteres Titrationsexperiment durchgeführt. In Abb. 7 ist zu erkennen, dass die Elektrode trotz der Biegung über den Versuchszeitraum von ca. 5000 s stabile Messwerte liefert und auf die Änderungen des pH-Werts sowohl bei Zugabe von $\mathrm{HCl}$ als auch bei Zugabe von $\mathrm{KOH}$ reagiert. Die ermittelte Empfindlichkeit liegt erneut bei ca. $-70 \mathrm{mV} / \mathrm{pH}$ und ist
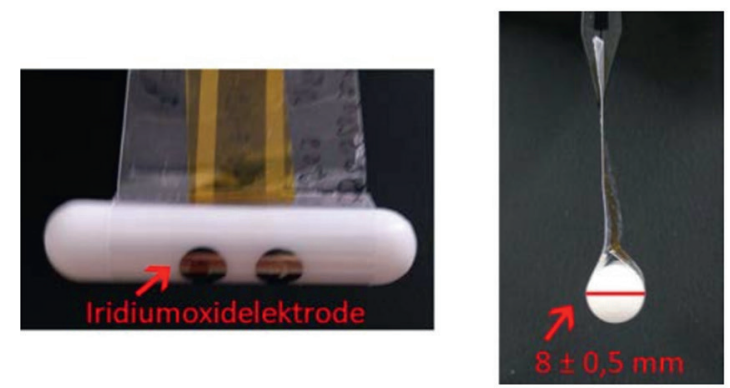

Abb. 6: Frontal- (links) und Seitenansicht (rechts) einer gebogenen flexiblen Elektrode. 


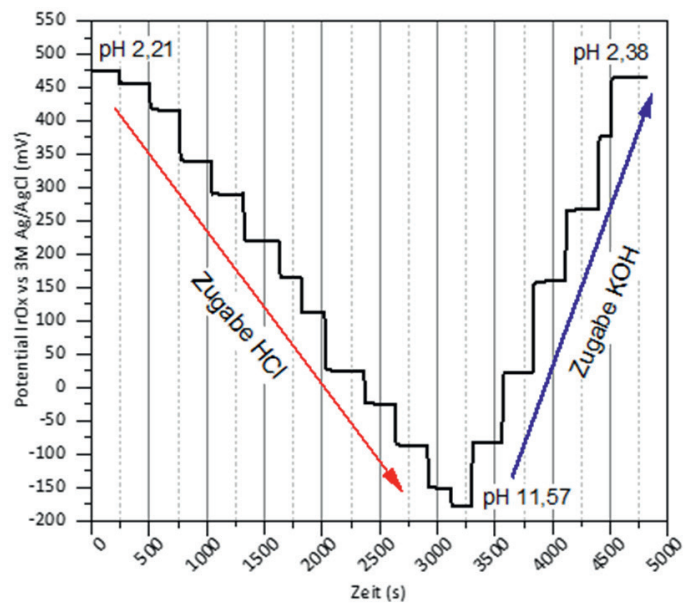

Abb. 7: Sensorverhalten einer gebogenen IrOx-pH-Elektrode über die Zeit bei Titration mit $\mathrm{HCl}$ und $\mathrm{KOH}$ gegenüber der kommerziellen Referenzelektrode.

damit vergleichbar mit den Ergebnissen für die nicht gebogene Elektrode (vgl. Abb. 4).

Die bisher gezeigten Ergebnisse wurden mit einer externen Referenzelektrode $(\mathrm{Ag} / \mathrm{AgCl} 3 \mathrm{M} \mathrm{KCl})$ bestimmt. Die Sensoreigenschaften des hergestellten Sensors als Kombination aus integrierter QRE und IrOx-Elektrode sind in Abb. 8 gezeigt. In verschiedenen Pufferlösungen, deren Chloridionenkonzentration konstant gehalten wurde, zeigt sich eine Sensorantwort mit einer Empfindlichkeit von ca. $-66 \mathrm{mV} / \mathrm{pH}$ und einer leichten Drift. Es ist zu vermuten, dass die etwas geringere Empfindlichkeit und auch die Drift wenigstens teilweise auf die QRE zurückzuführen sind. Möglicherweise gehen Teile des in der aufgerakelten Paste enthaltenen Silbers oder auch Silberchlorids in Lösung und beeinflussen somit das Potential und die Potentialstabilität. Nichtsdestotrotz eröffnet das in Abb. 8 gezeigte Ergebnis eine Vielzahl möglicher Anwendungen der hergestellten flexiblen $\mathrm{pH}$-Sensoren.

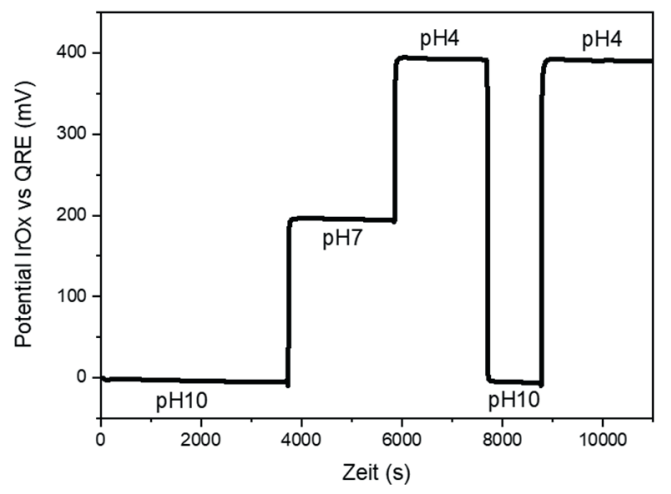

Abb. 8: Gemessenes Potential zwischen integrierter QRE und IrOx-Elektrode bei verschiedenen $\mathrm{pH}-$ Werten.

\section{Zusammenfassung und Ausblick}

Im Rahmen der Untersuchungen wurde ein neues Konzept für die Realisierung eines ultra-dünnen und flexiblen potentiometrischen $\mathrm{pH}$-Sensor erfolgreich demonstriert. Dabei werden Empfindlichkeiten der Arbeitselektrode von bis $\mathrm{zu}-73 \mathrm{mV} / \mathrm{pH}$ im $\mathrm{pH}-$ Bereich zwischen $\mathrm{pH} 2$ und $\mathrm{pH}$ 11,5 erreicht. Gleichzeitig besitzt der Sensor nur eine geringe Driftrate von weniger als $1 \mathrm{mV} / \mathrm{h}$. Insbesondere sind die Sensoreigenschaften dabei im flachen und gebogenen Zustand des Sensors vergleichbar. Der etablierte flexible $\mathrm{pH}-$ Sensor eröffnet damit eine Vielzahl an Anwendungen wie bspw. in smarten Klebebändern oder zur Überwachung von Korrosion. Da sowohl Substratmaterial als auch die Materialien für die Arbeitselektrode biokompatibel sind, können mit dem flexiblen Sensor je nach Ausführung der Referenzelektrode sogar Anwendungen wie medizinische Wearables und Implantate adressiert werden, bei denen Biokompatibilität gefordert ist.

Zur Verbesserung der Sensoreigenschaften muss vor allem die QRE in eine vollwertige Referenzelektrode weiterentwickelt werden, um Messungen in verschiedenen Medien unabhängig von der Chloridionenkonzentration durchführen zu können. Hierzu kann z.B. eine salzbeladene Polymerschicht über der Elektrode aufgebracht werden. Die Arbeiten dazu bauen auf ersten Versuchen aus früheren Arbeiten auf [6].

\section{Literatur}

[1] SELBMANN, F. ; BAUM, M. ; WIEMER, M. ; GESSNER, T.: Deposition of Parylene $C$ and Characterization of its Hermeticity for the Encapsulation of MEMS and Medical Devices. In: Tagungsband $11^{\text {th }}$ IEEE Annual International Conference on Nano/Micro Engineered and molecular Systems, Matsushima Bay und Sendai / Japan, April 2016

[2] SELBMANN, F. ; BAUM, M. ; MEINECKE, C. ; WIEMER, M. ; KUHN, H. ; JOSEPH, Y.: Low Temperature Parylene Based Adhesive Bonding Technology for $150 \mathrm{~mm}$ and $200 \mathrm{~mm}$ Wafers for Fully Biocompatible and Highly Reliable Microsystems. In: ECS J. Solid State Sci. Technol. 10 (2021) 074010

[3] SELBMANN, F. ; ROSCHER, F. ; TORTATO, F. d. S. ; WIEMER, M. ; OTTO, T. ; JOSEPH, Y.: An ultra-thin and highly flexible multilayer Printed Circuit Board based on Parylene. In: Tagungsband Smart Systems Integration, online, März 2021

[4] MARZOUK, S.A.M.: Improved Electrodeposited Iridium Oxide $\mathrm{pH}$ Sensor Fabricated on Etched Titanium Substrates. In: Analytic Chemistry 75 (2003) 1258-1266

[5] MIERSCH, C.: Entwicklung, Herstellung und Charakterisierung eines flexiblen $\mathrm{pH}$-Sensors auf Parylene-CSubstrat, Technische Universität Bergakademie Freiberg, Masterarbeit, 2019

[6] GÜTH, F. ; ARKI, P. ; LÖHER, T. ; OSTMANN, A. ; JOSEPH, Y. : Electrochemical Sensors Based on Printed Circuit Board Technologies. In: Procedia Engineering 168 (2016) 452-455 


\section{Danksagung}

Unser Dank gilt allen beteiligten Kolleginnen und Kollegen des Instituts für Elektronik- und Sensormaterialien, des Instituts für Angewandte Physik, sowie des Instituts für Werkstoffwissenschaften der TU Bergakademie Freiberg. Ein besonderer Dank geht dabei an Valentin Garbe und Florian Honeit für das Sputtern, Susann Rabe für die Herstellung der Querschliffe sowie Brigitte Bleiber für die Anfertigung der REM-Aufnahmen. Ein weiterer besonderer Dank gebührt dem Fraunhofer-Institut für Elektronische Nanosysteme ENAS für die Bereitstellung der freistehenden flexiblen Parylene-Substrate. 